\title{
パラメータを外乱と見なした非線形適応 $\mathcal{H}_{\infty}$ 制御系の 構成法 ある種の非線形パラメトリックモデルに対する適用*
}

\author{
Nonlinear Adaptive $\mathcal{H}_{\infty}$ Control Systems for Bounded \\ Variations of Parameters \\ - Nonlinear Parametric Model*
}

Yoshihiko MiYasato $^{\dagger}$

\begin{abstract}
A new class of adaptive nonlinear $\mathcal{H}_{\infty}$ control systems for nonlinear and time-varying processes which include nonlinear parametric models approximated by neural networks (NN), is proposed in this manuscript. Those control schemes are derived as solutions of particular nonlinear $\mathcal{H}_{\infty}$ control problems, where unknown system parameters, approximation and algorithmic errors in NN, and estimation errors of layer weights in NN, are regarded as exogenous disturbances to the processes, and thus, in the resulting control systems, the $\mathcal{L}_{2}$ gains from those uncertain elements to generalized outputs are made less than $\gamma(>0)$ (the prescribed positive constants). The resulting control systems are bounded for arbitrarily large but bounded variations of time-varying parameters and layer weights, and modeling and algorithmic errors in NN approximators.
\end{abstract}

\section{1. 緒言}

ニューラルネット（Neural Network : NN）の関数近 似能力に着目して，NNを非線形制御や非線形適応制御 に適用する試みはこれまでに多くある。しかしNNは非 線形関数の表現の誤差 (近似誤差) に加えて, 各層の重 み係数の学習が $\mathrm{NN}$ の線形回帰モデルに基づいて行われ る(誤差逆伝搬法) という算法上の誤差も存在するため, 大域的な安定解析は困難であり, 制御系の構成に関して 厳密な解析に基づく結果は得られていなかった [1-4].こ れに対し近年, 従来とは異なる線形回帰モデルに対する 算法上の誤差の厳密な評価が可能になり [5], この評価 と結びついた安定解析に基づく NNのロバスト非線形適 応制御も提案されつつある [5-7]. そこでは過渡特性な どの制御性能に関する議論も行われているが, 最適性と 関係づけた解析には至っていない.

これに対して本論文では， $\mathcal{H}_{\infty}$ 制御の規範に基づいて, NNを用いた非線形適応制御系の構成法について論じる. 提案する手法では, システムパラメータの時間変動成分, $\mathrm{NN}$ の近似䛊差と算法上の誤差, および $\mathrm{NN}$ の各層の重

\footnotetext{
* 原稿受付 2004 年 1 月 9 日

$\dagger$ 統計数理研究所 The Institute of Statistical Mathematics; Minami-Azabu, Minato-ku, Tokyo 106-8569, JAPAN

Key Words: adaptive control, nonlinear control, $\mathcal{H}_{\infty}$ control, neural network.
}

み係数の推定誤差をシステムに加わる等価的な外乱と見 なして, その外乱から一般化出力 (制御誤差と入力信号 の一部) への $\mathcal{L}^{2}$ ゲインを規定する非線形 $\mathcal{H}_{\infty}$ 制御系を 逆最適性の立場から構成する [8-13]. その結果得られる 制御系においては，非線形特性やシステムパラメータが 時間変化する場合でも制御系全体の有界性が保たれ，ま た等価的な外乱から一般化出力への $\mathcal{L}^{2}$ ゲインを規定す るパラメータを小さく設定することで, NN の関数近似 䛊差の大きさに関わらずに，制御誤差を任意に小さくす ることができる。ささらにシステムの特性が時間不変で, $\mathrm{NN}$ の近似誤差と算法上の誤差が存在しない理想的な場 合には，制御誤差の零収束性も保証される。これらの事 柄が安定解析だけではなく, 特定の指標に対する最適性 と関連づけて議論できるところに提案する手法の特徴が ある。

\section{2. 問題設定}

相対次数が $n^{*}$ 次の 1 入力 1 出力非線形系を考える.

$$
\begin{aligned}
\frac{d}{d t} e(t)= & f_{1}(e(t), r(t))+f_{2}(e(t), r(t)) \\
& +b_{0} u_{f n^{*}-1}(t)+\Phi_{1}^{\mathrm{T}} \omega_{1}(t) \\
u_{f i}(t)= & \frac{1}{(s+\lambda)^{i}} u(t) \quad(\lambda>0)
\end{aligned}
$$

ただし $e(t)$ は制御変数 (出力值または追従誤差など), $u(t)$ は制御入力, $r(t)$ は有界な外生信号, $f_{1}(e(t), r(t))$, 
$f_{2}(e(t), r(t))$ は未知の非線形項とする.とくに $f_{1}(e, r)$ は 構造が規定できる非線形項 (NN で近似できる), $f_{2}(e, r)$ は外乱も含めた構造の規定できない非線形項とする. $\omega_{1}(t)$ は測定可能な信号から構成されるべクトル, $b_{0}$ と $\Phi_{1}$ は未知のシステムパラメータ（時変パラメータでもよ い）とする。次の仮定をおく。

（仮定 1） 1） $b_{0}$ は時変の場合も考えるが，その符号 は不変で既知とする．以後では一般性を失うことなく $b_{0}>0$ とする.

2) 未知の非線形要素 $f_{2}(e, r)$ は次のように評価される.

$$
\left|f_{2}(e, r)\right| \leq d_{2} \cdot \phi_{2}(e, r)
$$

ただし $d_{2}$ は未知の正の定数で， $\phi_{2}(e, r)(>0)$ は既知の 関数とする.

3) $\omega_{1}(t)$ は次のように評価される $[12]$.

$$
\left\|\omega_{1}(t)\right\| \leq N_{1} \cdot \sup _{t \geq \tau}|e(\tau)|+N_{2}\left(N_{1}, N_{2}>0\right)
$$

また $\dot{\omega}_{1}(t) \sim \omega_{1}^{\left(n^{*}-1\right)}(t)$ は測定可能な信号ベクトルであ り以下のように評価される。

$$
\begin{aligned}
\left\|\omega_{1}^{(i)}(t)\right\| \leq & N_{3} \cdot \sup _{t \geq \tau}|e(\tau)|+N_{4} \cdot \sup _{t \geq \tau}\left|u_{f n^{*}-i}(\tau)\right| \\
& +N_{5} \\
& \left(N_{3} \sim N_{5}>0\right), \quad\left(1 \leq i \leq n^{*}-1\right)
\end{aligned}
$$

4) パラメータ $\Phi_{1}$ のノルムの上界 $\left(M_{1}\right)$ が既知とする. また高調波利得 $b_{0}$ の上界 $\bar{b}_{0}$ と下界 $\underline{b}_{0}$ を評価する $\delta_{b}, M_{b}$ も既知であり

$$
0<\delta_{b} \leq \underline{b}_{0} \leq b_{0} \leq \bar{b}_{0} \leq M_{b}<\infty
$$

以下の $p$ の上界 $\bar{p}$ と下界 $\underline{p}$ に関しても同様の $\delta_{p}, M_{p}$ が 既知とする。

$$
\begin{aligned}
& p \equiv \frac{1}{b_{0}}, \bar{p} \equiv \frac{1}{\underline{b}_{0}}, \underline{p} \equiv \frac{1}{\bar{b}_{0}} \\
& 0<\delta_{p} \leq \underline{p} \leq p \leq \bar{p} \leq M_{p}<\infty
\end{aligned}
$$

以上のような設定のもとで, 次の性質を有する非線形適 応制御系を導出する。

1.（安定性の条件）任意の有界な時変パラメー夕に対 して制御系は有界となる。またとくに時不変パラメータ または時変パラメー夕が一定值に収束し，NNに含まれ る誤差が 0 の時には, 制御変数 $e(t)$ の漸近安定性が保 証される。

2.（最適性の条件）得られた制御系が特定の制御指標 に対して，最適または準最適となる。

【注意 1】(1), (2) 式の制御対象は，いくつかの適応 制御問題を含む一般的な表現形式となっている $[12,13]$ （たとえば文献 [12] の付録 A 参照）。また仮定 1-3 はその ような適応制御問題において制御対象の最小位相性に対 応している（文献 [12] の付録 B 参照）。

\section{3. 非線形パラメトリックモデル}

非線形成分 $f_{1}(e, r)$ を 3 層の $\mathrm{NN}$ の出力 $g(z)$ で近似 する。

$$
\begin{aligned}
& g(z)=W^{\mathrm{T}} S\left(V^{\mathrm{T}} \bar{Z}\right) \in \mathbf{R} \\
& S\left(V^{\mathrm{T}} \bar{Z}\right)=\left[s\left(v_{1}^{\mathrm{T}} \bar{Z}\right), \cdots, s\left(v_{m}^{\mathrm{T}} \bar{Z}\right)\right]^{\mathrm{T}} \in \mathbf{R}^{m} \\
& \bar{Z}=\left[z^{\mathrm{T}}, 1\right]^{\mathrm{T}}=[e, r, 1]^{\mathrm{T}} \in \mathbf{R}^{3}, z=[e, r]^{\mathrm{T}} \in \mathbf{R}^{2} \\
& s(x)=\frac{1}{1+\exp (-\gamma x)}(\gamma>0) \\
& W=\left[w_{1}, \cdots, w_{m}\right]^{\mathrm{T}} \in \mathbf{R}^{m} \\
& V=\left[v_{1}, \cdots, v_{m}\right] \in \mathbf{R}^{3 \times m} \quad\left(v_{i} \in \mathbf{R}^{3}\right)
\end{aligned}
$$

$\mathrm{NN}$ のユニット数 $(m)$ を十分多くとることで $g(z)$ が $f_{1}(e, r)$ を十分よく近似して $[14]$, 次の仮定 2 が成立する ものとする.

(仮定 2） 次式を満たす重み係数 $V$ と $W$ （時変も含 む）が存在する。

$$
f_{1}(e, r)=g(z)+\mu_{1},\left|\mu_{1}\right| \leq d_{1} \cdot \phi_{1}(e, r)
$$

ただし $d_{1}$ は未知の正の定数で, $\phi_{1}(e, r)(>0)$ は既知の 関数とする。またこの $V$ と $W$ のノルムの上界が既知と する.

$V$ と $W$ の推定值をそれぞれ $\hat{V}$ と $\hat{W}$ とおいて, 推定值を 逐次的に求める. この時, 推定モデル $\hat{g}(z)=\hat{W}^{\mathrm{T}} S\left(\hat{V}^{\mathrm{T}} \bar{Z}\right)$ と真のモデル $g(z)$ の間に次のような関係式が成立する $[5,15]$.

【補題 1】 $\hat{g}(z)$ と $g(z)$ の誤差は次のように評価さ れる。

$$
\begin{aligned}
& \hat{g}(z)-g(z)=\hat{W}^{\mathrm{T}} S\left(\hat{V}^{\mathrm{T}} \bar{Z}\right)-W^{\mathrm{T}} S\left(V^{\mathrm{T}} \bar{Z}\right) \\
& \quad=\tilde{W}^{\mathrm{T}}\left(\hat{S}-\hat{S}^{\prime} \hat{V}^{\mathrm{T}} \bar{Z}\right)+\hat{W}^{\mathrm{T}} \hat{S}^{\prime} \tilde{V}^{\mathrm{T}} \bar{Z}+\mu_{2} \\
& \tilde{W}=\hat{W}-W, \tilde{V}=\hat{V}-V \\
& \hat{S}=S\left(\hat{V}^{\mathrm{T}} \bar{Z}\right) \\
& \hat{S}^{\prime}=\operatorname{diag}\left(\hat{s}_{1}^{\prime}, \cdots, \hat{s}_{m}^{\prime}\right) \\
& \hat{s}_{i}^{\prime}=s^{\prime}\left(\hat{v}_{i}^{\mathrm{T}} \bar{Z}\right)=\left[\frac{d s(z)}{d z}\right]_{z=\hat{v}_{i}^{\mathrm{T}} \bar{Z}} \\
& \left|\mu_{2}\right| \leq\|V\| \cdot\left\|\bar{Z} \hat{W}^{\mathrm{T}} \hat{S}^{\prime}\right\|+\|W\| \cdot\left\|\hat{S}^{\prime} \hat{V}^{\mathrm{T}} \bar{Z}\right\|+|W|_{1}
\end{aligned}
$$

【注意 2】 $\mu_{2}$ を直接に表すと以下のようになる。

$$
\mu_{2}=\tilde{W}^{\mathrm{T}} \hat{S}^{\prime} V^{\mathrm{T}} \bar{Z}+\mathcal{O}\left\{\left(\tilde{V}^{\mathrm{T}} \bar{Z}\right)^{2}\right\}
$$

つまり $\mu_{2}$ を評価する式は $\tilde{W}=0, \tilde{V}=0$ で 0 にならない が， $\mu_{2}$ そのものは $\tilde{W}=0, \tilde{V}=0$ で 0 になる。 また通常 の誤差逆伝搬法で用いられる線形回帰モデルは次式の部 分である。

$$
\tilde{W}^{\mathrm{T}} \hat{S}+\hat{W}^{\mathrm{T}} \hat{S}^{\prime} \tilde{V}^{\mathrm{T}} \bar{Z}
$$

【注意 3】 NN の関数近似はコンパクトな領域で論じ られるが，ここでは大域的な領域 $\mathbf{R}^{2}$ で仮定 2 が成立す 
るものとする.このため重み係数 $W, V$ は一定の值とは 限らず，領域の変化に応じて変動するものとする。これ らは時間変動の重み係数として捉えることができる．ま た $f_{1}(e, r)$ の大域的な挙動は $\mu_{1}$ を評価する $\phi_{1}(e, r)$ にも 反映されているものとする（そのように $\phi_{1}(e, r)$ を決定 する）。

\section{4. 非線形適応 $\mathcal{H}_{\infty}$ 制御}

以下にバックステッピング $[16]$ に基づいて $n^{*}$ ステッ プ $\left(n^{*}+1\right.$ ステップ目は補助信号と適応則の決定）で構 成法を示す。

Step 1) $z_{1}(t)$ と $z_{2}(t)$ を次のように定めて

$$
\begin{aligned}
& z_{1}(t) \equiv e(t) \\
& z_{2}(t) \equiv u_{f n^{*}-1}(t)-\alpha_{1}(t)
\end{aligned}
$$

仮想入力 $\alpha_{1}(t)$ を以下のように決定する.

$$
\begin{aligned}
\alpha_{1}(t)= & -\hat{p}(t)\left\{\hat{\Phi}_{1}(t)^{\mathrm{T}} \omega_{1}(t)+\hat{W}(t)^{\mathrm{T}} S\left(\hat{V}(t)^{\mathrm{T}} \bar{Z}(t)\right)\right\} \\
& +v_{1}(t)+\tilde{\alpha}_{1}(t) \\
\equiv & -\hat{p}(t) v_{0}(t)+v_{1}(t)+\tilde{\alpha}_{1}(t)
\end{aligned}
$$

$v_{1}(t)$ と $\tilde{\alpha}_{1}(t)$ は後に定める信号である.また先の $g(z)$ と $\hat{g}(z)$ の誤差を次のように表す.

$$
\begin{aligned}
& \hat{g}(z)-g(z)=\tilde{W}^{\mathrm{T}}\left(\hat{S}-\hat{S}^{\prime} \hat{V}^{\mathrm{T}} \bar{Z}\right)+\hat{W}^{\mathrm{T}} \hat{S}^{\prime} \tilde{V}^{\mathrm{T}} \bar{Z}+\mu_{2} \\
& \quad=\tilde{W}^{\mathrm{T}} \omega_{20}+\sum_{i=1}^{m} \tilde{v}_{i}^{\mathrm{T}} \omega_{2 i}+\mu_{2} \equiv \tilde{\Phi}_{2}^{\mathrm{T}} \omega_{2}+\mu_{2} \\
& \omega_{20}=\hat{S}-\hat{S}^{\prime} \hat{V}^{\mathrm{T}} \bar{Z} \in \mathbf{R}^{m} \\
& \omega_{2 i}=\left(\hat{w}_{i} \hat{s}_{i}^{\prime}\right) \bar{Z} \in \mathbf{R}^{3} \quad(1 \leq i \leq m) \\
& \Phi_{2}=\left[W^{\mathrm{T}}, v_{1}^{\mathrm{T}}, \cdots, v_{m}^{\mathrm{T}}\right]^{\mathrm{T}} \in \mathbf{R}^{4 m} \\
& \hat{\Phi}_{2}=\left[\hat{W}^{\mathrm{T}}, \hat{v}_{1}^{\mathrm{T}}, \cdots, \hat{v}_{m}^{\mathrm{T}}\right]^{\mathrm{T}} \in \mathbf{R}^{4 m} \\
& \tilde{\Phi}_{2}=\hat{\Phi}_{2}-\Phi_{2}=\left[\tilde{W}^{\mathrm{T}}, \tilde{v}_{1}^{\mathrm{T}}, \cdots, \tilde{v}_{m}^{\mathrm{T}}\right]^{\mathrm{T}} \in \mathbf{R}^{4 m} \\
& \left(\tilde{v}_{i}=\hat{v}_{i}-v_{i}\right) \\
& \omega_{2}=\left[\omega_{20}^{\mathrm{T}}, \omega_{21}^{\mathrm{T}}, \cdots, \omega_{2 m}^{\mathrm{T}}\right]^{\mathrm{T}} \in \mathbf{R}^{4 m}
\end{aligned}
$$

調整パラメータ $\hat{p}(t)$ は射影型の適応則 [17]により決定 する。

$$
\dot{\hat{p}}(t)=\left\{\begin{array}{cl}
g_{3} v_{0} z_{1}(t) & \text { Case } \mathrm{I}_{p} \\
0 & \text { Case } \mathrm{II}_{p}
\end{array}\right.
$$

たたし $g_{3}>0$ で Case $\mathrm{I}_{p}, \mathrm{II}_{p}$ は次のように定める.

Case $\mathrm{I}_{p}: \delta_{p}<\hat{p}<M_{p}$, または $\hat{p}=\delta_{p} \& v_{0}(t) z_{1}(t) \geq 0$, または $\hat{p}=M_{p} \& v_{0}(t) z_{1}(t) \leq 0$

Case $\mathrm{II}_{p}$ ：その他の場合 これに対して正定関数 $V_{1}(t)$ を定めて（ただし $\Phi_{i}^{*}$ は時変 パラメータ $\Phi_{i}$ の代表值 (時不変) とする. とくに $\Phi_{i}$ が 時不変の時は $\Phi_{i}^{*}=\Phi_{i}$ となる.)

$$
V_{1}(t)=\frac{1}{2} z_{1}(t)^{2}
$$

$$
\begin{aligned}
& +\frac{1}{2} \sum_{i=1}^{2}\left\{\hat{\Phi}_{i}(t)-\Phi_{i}^{*}\right\}^{\mathrm{T}} G_{i}^{-1}\left\{\hat{\Phi}_{i}(t)-\Phi_{i}^{*}\right\} \\
& +\frac{\underline{b}_{0}}{2}\{\hat{p}(t)-\bar{p}\}^{2} / g_{3} \\
& +\frac{1}{2}\left\{\hat{b}_{0}(t)-\underline{b}_{0}\right\}^{2} / g_{4} \\
& \quad\left(G_{i}=G_{i}^{\mathrm{T}}>0, g_{4}>0\right)
\end{aligned}
$$

$V_{1}(t)$ の時間微分を計算する。

$$
\begin{aligned}
\dot{V}_{1}(t) \leq & \tilde{\Theta}^{\mathrm{T}} \tilde{\omega}_{1}(t) z_{1}(t)+f_{2}(e, r) z_{1}(t) \\
& +\left(\mu_{1}+\mu_{2}\right) z_{1}(t) \\
& +\left\{\hat{b}_{0}(t)+\tilde{b}_{0}(t)\right\} v_{1}(t) z_{1}(t) \\
& +b_{0} z_{1}(t) z_{2}(t)+b_{0} \tilde{\alpha}_{1}(t) z_{1}(t) \\
& +\sum_{i=1}^{2}\left\{\hat{\Phi}_{i}(t)-\Phi_{i}^{*}\right\}^{\mathrm{T}} G_{i}^{-1}\left\{\dot{\hat{\Phi}}_{i}(t)-\tau_{\phi_{i} 1}(t)\right\} \\
& +\sum_{i=1}^{2} \sigma_{i}\left(\left\|\hat{\Phi}_{i}\right\|\right)\left\{\Phi_{i}^{*}-\hat{\Phi}_{i}(t)\right\}^{\mathrm{T}} \hat{\Phi}_{i}(t) \\
& +\left\{\hat{b}_{0}(t)-\underline{b}_{0}\right\}\left\{\dot{\hat{b}}_{0}(t)-\tau_{b 1}(t)\right\} / g_{4} \\
\Theta \equiv & {\left[\left(\Phi_{1}-\Phi_{1}^{*}\right)^{\mathrm{T}},\left(\Phi_{2}-\Phi_{2}^{*}\right)^{\mathrm{T}}, \underline{b}_{0}-b_{0}\right]^{\mathrm{T}} } \\
\tilde{b}_{0} \equiv & b_{0}-\underline{b}_{0}(\geq 0) \\
\tilde{\omega}_{1}(t)= & {\left[\omega_{1}(t)^{\mathrm{T}}, \omega_{2}(t)^{\mathrm{T}}, \hat{p}(t) v_{0}(t)\right]^{\mathrm{T}} } \\
\tau_{\phi_{1} 1}(t)= & G_{1} \omega_{1}(t) z_{1}(t)-\sigma_{1}\left(\left\|\hat{\Phi}_{1}\right\|\right) G_{1} \hat{\Phi}_{1}(t) \\
\tau_{\phi_{2} 1}(t)= & G_{2} \omega_{2}(t) z_{1}(t)-\sigma_{2}\left(\left\|\hat{\Phi}_{2}\right\|\right) G_{2} \hat{\Phi}_{2}(t) \\
\tau_{b 1}(t)= & g_{4} v_{1}(t) z_{1}(t)
\end{aligned}
$$

ただし $\sigma_{i}\left(\left\|\hat{\Phi}_{i}\right\|\right)(i=1,2)$ は後に定める連続微分可能な 正值の関数とする. [13] と同様にして (35) 式右辺の第 1 項〜 4 項より次の仮想的なシステムを考え

$$
\begin{aligned}
& \dot{z}_{1}=f\left(z_{1}\right)+g_{11} d+g_{12} v_{1} \\
& f\left(z_{1}\right)=0 \\
& g_{11}=\left[\left\|\tilde{\omega}_{1}\right\|, \phi_{1}(e, r), \phi_{2}(e, r),\right. \\
& \left.\quad\left\|\bar{Z} \hat{W}^{\mathrm{T}} \hat{S}^{\prime}\right\|,\left\|\hat{S}^{\prime} \hat{V}^{\mathrm{T}} \bar{Z}\right\|, 1\right] \\
& \equiv \Omega_{1}\left(z_{1}, r, \tilde{\omega}_{1}\right)^{\mathrm{T}} \\
& d=\left[\|\tilde{\Theta}\|, d_{1}, d_{2},\|V\|,\|W\|,\|W\|_{1}\right]^{\mathrm{T}} \\
& \equiv \Delta\left(\Phi_{1}, \Phi_{2}, b_{0}\right) \\
& g_{12}=\hat{b}_{0}+\tilde{b}_{0}
\end{aligned}
$$

$d=\Delta\left(\Phi_{1}, \Phi_{2}, b_{0}\right)((45)$ 式）をシステムに加わる未知外 乱と考えて, 非線形 $\mathcal{H}_{\infty}$ 制御の手法で $v_{1}$ を用いてシス テムを安定化する．そのために正定数 $\gamma_{1}^{*}$ に対して正定 関数 $\tilde{V}_{1}$

$$
\tilde{V}_{1}(t)=\frac{1}{2} z_{1}(t)^{2}
$$

が次の Hamilton-Jacobi-Isaacs (HJI) 方程式の解とな るように正值の関数 $h_{1}$ と $r_{1}$ を求める.

$$
\frac{\partial \tilde{V}_{1}}{\partial z_{1}} f_{1}+\frac{1}{4}\left(\frac{\left\|g_{11}\right\|^{2}}{\gamma_{1}^{* 2}}-\frac{g_{12}^{2}}{r_{1}}\right)\left(\frac{\partial \tilde{V}_{1}}{\partial z_{1}}\right)^{2}+h_{1} z_{1}^{2} \leq 0
$$




$$
\Leftrightarrow\left\{\frac{\left\|\Omega_{1}\right\|^{2}}{\gamma_{1}^{* 2}}-\frac{\left(\hat{b}_{0}+\tilde{b}_{0}\right)^{2}}{r_{1}}\right\} \frac{z_{1}^{2}}{4}+h z_{1}^{2} \leq 0
$$

上式には未知の $\tilde{b}_{0}$ が含まれるので, $\tilde{b}_{0} \geq 0$ ((37) 式) を 考慮して次式を満足する $h_{1}$ と $r_{1}$ を求める（下記の式は 上式の十分条件）.

$$
\left(\frac{\|\left.\Omega_{1}\right|^{2}}{\gamma_{1}^{* 2}}-\frac{\hat{b}_{0}^{2}}{r_{1}}\right) \frac{z_{1}^{2}}{4}+h_{1} z_{1}^{2} \leq 0
$$

これより非線形 $\mathcal{H}_{\infty}$ 制御の解としての入力が以下のよう になるが

$$
v_{1}^{*}=-\frac{1}{2 r_{1}} g_{12} \frac{\partial \tilde{V}_{1}}{\partial z_{1}}=-\frac{1}{2 r_{1}}\left(\hat{b}_{0}+\tilde{b}_{0}\right) z_{1}
$$

上式には未知の $\tilde{b}_{0}$ が含まれるので, 以下の制御入力で代 用する。

$$
v_{1}^{*}=-\frac{\hat{b}_{0}}{2 r_{1}} z_{1}
$$

この時, 元の系と $V_{1}(t)$, および $v_{1}^{*}(t)$ に特定化しない $v_{1}(t)$ に対して, 次の関係式が得られる.

$$
\begin{aligned}
\dot{V}_{1}(t) \leq & r_{1}\left\{\frac{\hat{b}_{0}(t) z_{1}(t)}{2 r_{1}}+v_{1}(t)\right\}^{2} \\
& -\left\{h_{1} z_{1}(t)^{2}+r_{1} v_{1}(t)^{2}\right\} \\
& -\gamma_{1}^{* 2}\left\|\Delta-\frac{1}{2 \gamma_{1}^{* 2}} \Omega_{1}\left|z_{1}(t)\right|\right\|^{2}+\gamma_{1}^{* 2}\|\Delta\|^{2} \\
& +\tilde{b}_{0}(t) v_{1}(t) z_{1}(t) t+b_{0} z_{1}(t) z_{2}(t)+b_{0} \tilde{\alpha}_{1}(t) z_{1}(t) \\
& +\sum_{i=1}^{2}\left\{\hat{\Phi}_{i}(t)-\Phi_{i}^{*}\right\}^{\mathrm{T}} G_{i}^{-1}\left\{\dot{\hat{\Phi}}_{i}(t)-\tau_{\phi_{i} 1}(t)\right\} \\
& +\sum_{i=1}^{2} \sigma_{i}\left(\left\|\hat{\Phi}_{i}\right\|\right)\left\{\Phi_{i}^{*}-\hat{\Phi}_{i}(t)\right\}^{\mathrm{T}} \hat{\Phi}_{i}(t) \\
& +\left\{\hat{b}_{0}(t)-\underline{b}_{0}\right\}\left\{\dot{\hat{b}}_{0}(t)-\tau_{b 1}(t)\right\} / g_{4}
\end{aligned}
$$

Step i) $\left(2 \leq i \leq n^{*}\right) \quad z_{i}(t)$ の時間微分を計算する.

$$
\begin{aligned}
z_{i}(t) \equiv & u_{f n^{*}-i+1}(t)-\alpha_{i-1}(t) \\
\dot{z}_{i}(t)= & -\lambda u_{f n^{*}-i+1}(t)+u_{f n^{*}-i}(t)-\beta_{i-1}(t) \\
& -\gamma_{i-1}(t)\left\{f_{1}\left(z_{1}, r\right)+f_{2}\left(z_{1}, r\right)\right. \\
& \left.\quad+b_{0} u_{f n^{*}-1}(t)+\Phi_{1}^{\mathrm{T}} \omega_{1}(t)\right\} \\
& -\gamma_{p i-1}(t) \dot{\hat{p}}(t)-\gamma_{\phi_{1} i-1}(t) \dot{\hat{\Phi}}_{1}(t) \\
& -\gamma_{\phi_{2} i-1}(t) \dot{\hat{\phi}}_{2}(t)-\gamma_{b i-1}(t) \dot{\hat{b}}_{0}(t) \\
\beta_{i-1}(t)= & \frac{\partial \alpha_{i-1}}{\partial \omega_{(i-1)}} \dot{\omega}_{(i-1)}(t) \\
& +\sum_{j=2}^{i-1} \frac{\partial \alpha_{i-1}}{\partial z_{j}}\left\{-\lambda u_{f n^{*}-j+1}(t)\right. \\
& \left.\quad+u_{f n^{*}-j}(t)-\beta_{j-1}(t)\right\} \\
\gamma_{i-1}(t)= & \frac{\partial \alpha_{i-1}}{\partial z_{1}}-\sum_{j=2}^{i-1} \frac{\partial \alpha_{i-1}}{\partial z_{j}} \gamma_{j-1}(t)
\end{aligned}
$$

$$
\begin{aligned}
& \gamma_{p i-1}(t)=\frac{\partial \alpha_{i-1}}{\partial \hat{p}}-\sum_{j=2}^{i-1} \frac{\partial \alpha_{i-1}}{\partial z_{j}} \gamma_{p j-1}(t) \\
& \gamma_{\phi_{1} i-1}(t)=\frac{\partial \alpha_{i-1}}{\partial \hat{\Phi}_{1}}-\sum_{j=2}^{i-1} \frac{\partial \alpha_{i-1}}{\partial z_{j}} \gamma_{\phi_{1} j-1}(t) \\
& \gamma_{\phi_{2} i-1}(t)=\frac{\partial \alpha_{i-1}}{\partial \hat{\Phi}_{2}}-\sum_{j=2}^{i-1} \frac{\partial \alpha_{i-1}}{\partial z_{j}} \gamma_{\phi_{2} j-1}(t) \\
& \gamma_{b i-1}(t)=\frac{\partial \alpha_{i-1}}{\partial \hat{b}_{0}}-\sum_{j=2}^{i-1} \frac{\partial \alpha_{i-1}}{\partial z_{j}} \gamma_{b j-1}(t) \\
& \omega_{(i-1)}(t)=\left\{u_{f n^{*}-1}(t) \sim u_{f n^{*}-i+2}, r, \dot{r}, \sim r^{(i-2)},\right. \\
& \omega_{(i-2)}(t), \dot{\omega}_{(i-2)}(t) \text { の独立な成分から } \\
& \text { 成る信号ベクトル }\}
\end{aligned}
$$

ただし $\sum_{j=2}^{1}(\cdot)$ の場合はこの項を加えないものとする. $z_{i}(t)$ に対して, $z_{i+1}(t)$ と $\alpha_{i}(t)$ を以下のように定める. ただ $v_{i}(t)$ と $\tilde{\alpha}_{i}(t)$ は後に定める信号である.

$$
\begin{aligned}
z_{i+1}(t) \equiv & u_{f n^{*}-i}(t)-\alpha_{i}(t) \\
\alpha_{i}(t)= & \lambda u_{f n^{*}-i+1}(t)+\beta_{i-1}(t)-\hat{c}_{i}(t) z_{i-1}(t) \\
& +\gamma_{i-1}(t)\left\{\hat{\Phi}_{1}(t)^{\mathrm{T}} \omega_{1}(t)\right. \\
& \left.\quad+\hat{W}(t)^{\mathrm{T}} S\left(\hat{V}(t)^{\mathrm{T}} \bar{Z}(t)\right)\right\} \\
& +\hat{b}_{0}(t) \gamma_{i-1}(t) u_{f n^{*}-1}(t)+v_{i}(t)+\tilde{\alpha}_{i}(t) \\
& +\gamma_{\phi_{1} i-1}(t) \tau_{\phi_{1} i}(t)+\gamma_{\phi_{2} i-1}(t) \tau_{\phi_{2} i}(t)(64) \\
\hat{c}_{i}(t)= & \left\{\begin{array}{cc}
\hat{b}_{0}(t) & (i=2) \\
1 & (i \geq 3)
\end{array}\right.
\end{aligned}
$$

これに対して正定関数 $V_{i}(t)$ を定め

$$
V_{i}(t)=\frac{1}{2} z_{i}(t)^{2}
$$

時間微分を計算すると, 先と同様にして次の関係が得ら れる.

$$
\begin{aligned}
& c_{i} z_{i-1}(t) z_{i}(t) \\
& +\sum_{j=1}^{2}\left\{\hat{\Phi}_{j}(t)-\Phi_{j}^{*}\right\}^{\mathrm{T}} G_{j}^{-1}\left\{\dot{\hat{\Phi}}_{j}(t)-\tau_{\phi_{j} i-1}(t)\right\} \\
& +\left\{\hat{b}_{0}(t)-\underline{b}_{0}\right\}\left\{\dot{\hat{b}}_{0}(t)-\tau_{b i-1}(t)\right\} t / g_{4}+\dot{V}_{i}(t) \\
& =\tilde{\Theta}^{\mathrm{T}} \tilde{\omega}_{i}(t) z_{i}(t)+z_{i}(t) z_{i+1}(t)+\gamma_{i-1}(t)\left(\mu_{1}+\mu_{2}\right) z_{i}(t) \\
& -\gamma_{i-1}(t) f_{2}\left(z_{1}, r\right) z_{i}(t)+z_{i}(t)\left\{v_{i}(t)+\tilde{\alpha}_{i}(t)\right\} \\
& +\sum_{j=1}^{2} z_{i}(t) \gamma_{\phi_{j} i-1}(t)\left\{\tau_{\phi_{j} i}(t)-\dot{\hat{\Phi}}_{j}(t)\right\} \\
& -\gamma_{p i-1}(t) \dot{\hat{p}}(t) z_{i}(t)-\gamma_{b i-1}(t) \dot{\hat{b}}_{0}(t) z_{i}(t) \\
& +\sum_{j=1}^{2}\left\{\hat{\Phi}_{j}(t)-\Phi_{j}^{*}\right\}^{\mathrm{T}} G_{j}^{-1}\left\{\dot{\hat{\Phi}}_{j}(t)-\tau_{\phi_{j} i}(t)\right\} \\
& +\left\{\hat{b}_{0}(t)-b_{0}^{*}\right\}\left\{\dot{\hat{b}_{0}}(t)-\tau_{b i}(\tau)\right\} / g_{4} \\
& \tau_{\phi_{1} i}(t)=\tau_{\phi_{1} i-1}(t)-G_{1} \gamma_{i-1}(t) \omega_{1}(t) z_{i}(t) \\
& \tau_{\phi_{2} i}(t)=\tau_{\phi_{2} i-1}(t)-G_{2} \gamma_{i-1}(t) \omega_{2}(t) z_{i}(t) \\
& \tau_{b i}(t)=\tau_{b i-1}(t)-g_{4} \gamma_{i-1}(t) u_{f n^{*}-1}(t) z_{i}(t) \\
& \tilde{\omega}_{i}(t)=\left[-\gamma_{i-1}(t) \omega_{1}(t)^{\mathrm{T}},-\gamma_{i-1}(t) \omega_{2}(t)^{\mathrm{T}},\right.
\end{aligned}
$$




$$
\begin{gathered}
\left.\gamma_{i-1}(t) u_{f n^{*}-1}(t),\right]^{\mathrm{T}} \\
c_{i}=\left\{\begin{array}{cl}
b_{0} & (i=2) \\
1 & (i \geq 3)
\end{array}\right.
\end{gathered}
$$

[13] と同様にして (67) 式右辺の第 1 項, 3 項, 4 項, 5 項 より次の仮想的なシステムを考え

$$
\begin{aligned}
& \dot{z}_{i}=f\left(z_{i}\right)+g_{i 1} d+g_{i 2} v_{i} \\
& f\left(z_{i}\right)=0 \\
& g_{i 1}=\left[\left\|\tilde{\omega}_{i}\right\|,\left|\gamma_{i-1}\right| \phi_{1}(e, r),\left|\gamma_{i-1}\right| \phi_{2}(e, r)\right. \\
& \left.\quad\left|\gamma_{i-1}\right|\left\|\bar{Z} \hat{W}^{\mathrm{T}} \hat{S}^{\prime}\right\|,\left|\gamma_{i-1}\right||| \hat{S}^{\prime} \hat{V}^{\mathrm{T}} \bar{Z} \|,\left|\gamma_{i-1}\right|\right] \\
& \quad \equiv \Omega_{i}\left(z_{1}, \cdots, z_{i}, r, \tilde{\omega}_{i}\right)^{\mathrm{T}} \\
& g_{i 2}=1
\end{aligned}
$$

$d=\Delta\left(\Phi_{1}, \Phi_{2}, b_{0}\right)((45)$ 式）をシステムに加わる外乱と 考えて, 非線形 $\mathcal{H}_{\infty}$ 制御の手法で $v_{i}$ を用いてシステムを 安定化する.このために正定数 $\gamma_{i}^{*}$ に対して正定関数 $\tilde{V}_{i}$

$$
\tilde{V}_{i}(t)=V_{i}(t)=\frac{1}{2} z_{i}(t)^{2}
$$

が次の HJI 方程式の解となるように正值の関数 $h_{i}$ と $r_{i}$ を求める.

$$
\begin{gathered}
\frac{\partial \tilde{V}_{i}}{\partial z_{i}} f_{i}+\frac{1}{4}\left(\frac{\left\|g_{i 1}\right\|^{2}}{\gamma_{i}^{* 2}}-\frac{g_{i 2}^{2}}{r_{i}}\right)\left(\frac{\partial \tilde{V}_{i}}{\partial z_{i}}\right)^{2}+h_{i} z_{i}^{2} \leq 0 \\
\Leftrightarrow\left\{\frac{\left\|\Omega_{i}\right\|^{2}}{\gamma_{i}^{* 2}}-\frac{1}{r_{i}}\right\} \frac{z_{i}^{2}}{4}+h_{i} z_{i}^{2} \leq 0
\end{gathered}
$$

これより非線形 $\mathcal{H}_{\infty}$ 制御の解としての入力が以下のよう に求まる.

$$
v_{i}^{*}=-\frac{1}{2 r_{i}} g_{i 2} \frac{\partial \tilde{V}_{i}}{\partial z_{i}}=-\frac{1}{2 r_{i}} z_{i}
$$

この時, 元の系と $V_{1}(t) \sim V_{i}(t)$, および $v_{1}^{*}(t) \sim v_{i}^{*}(t)$ に 特定化しない $v_{1}(t) \sim v_{i}(t)$ に対して次の関係式が得ら れる。

$$
\begin{aligned}
\sum_{j=1}^{i} \dot{V}_{j}( & \leq r_{1}\left\{\frac{\hat{b}_{0}(t) z_{1}(t)}{2 r_{1}}+v_{1}(t)\right\}^{2} \\
& -\left\{h_{1} z_{1}(t)^{2}+r_{1} v_{1}(t)^{2}\right\} \\
& -\gamma_{1}^{* 2}\left\|\Delta-\frac{1}{2 \gamma_{1}^{* 2}} \Omega_{1}(t)\left|z_{1}(t)\right|\right\|^{2}+\gamma_{1}^{* 2}\|\Delta\|^{2} \\
& +\tilde{b}_{0}(t) v_{1}(t) z_{1}(t) \\
& +\sum_{j=2}^{i}\left[r_{j}\left\{\frac{z_{j}(t)}{2 r_{j}}+v_{j}(t)\right\}^{2}\right. \\
& -\left\{h_{j} z_{j}(t)^{2}+r_{j} v_{j}(t)^{2}\right\} \\
& \left.-\gamma_{j}^{* 2}\left\|\Delta-\frac{1}{2 \gamma_{j}^{* 2}} \Omega_{j}(t) \mid z_{j}(t)\right\|^{2}+\gamma_{j}^{* 2}\|\Delta\|^{2}\right] \\
& +z_{i}(t) z_{i+1}(t)+\sum_{j=2}^{i} \gamma_{\phi_{1} j-1}(t)\left\{\tau_{\phi_{1} j}-\dot{\hat{\Phi}}_{1}(t)\right\}
\end{aligned}
$$

$$
\begin{aligned}
& +\sum_{j=2}^{i} \gamma_{\phi_{2} j-1}(t)\left\{\tau_{\phi_{2} j}-\dot{\hat{\Phi}}_{2}(t)\right\} \\
& -\sum_{j=2}^{i} \gamma_{p j-1}(\tau) \dot{\hat{p}}(t) z_{j}(t)-\sum_{j=2}^{i} \gamma_{b j-1}(t) \dot{\hat{b}}_{0}(t) z_{j}(t) \\
& +\sum_{j=1}^{2}\left\{\hat{\Phi}_{j}(t)-\Phi_{j}^{*}\right\}^{\mathrm{T}} G_{j}^{-1}\left\{\dot{\hat{\Phi}}_{j}(t)-\tau_{\phi_{j}}(t)\right\} \\
& +\sum_{j=1}^{2} \sigma_{j}\left(\left\|\hat{\Phi}_{j}\right\|\right)\left\{\Phi_{j}^{*}-\hat{\Phi}_{j}(t)\right\}^{\mathrm{T}} \hat{\Phi}_{j}(t) \\
& +\left\{\hat{b}_{0}(t)-\underline{b}_{0}\right\}\left\{\dot{\hat{b}}_{0}(t)-\tau_{b i}(t)\right\} / g_{4} \\
& +b_{0} \tilde{\alpha}_{1}(t) z_{1}(t)+\sum_{j=2}^{i} \tilde{\alpha}_{j}(t) z_{j}(t)
\end{aligned}
$$

最後の Step $\left.n^{*}\right)$ で実際の制御入力 $u(t)$ が決定される.

$$
u(t)=\alpha_{n^{*}}(t)
$$

Step $\left.\mathbf{n}^{*}+1\right) \quad \hat{\Phi}_{1}(t), \hat{\Phi}_{2}(t), \hat{b}_{0}(t), \tilde{\alpha}_{i}(t)$ の決定 $\hat{\Phi}_{1}(t), \hat{\Phi}_{2}(t), \hat{b}_{0}(t)$ の適応則と補助信号 $\tilde{\alpha}_{i}(t)$ は次の ように定める.

$$
\begin{aligned}
& \dot{\hat{\Phi}}_{1}(t)=\tau_{\phi_{1} n^{*}}(t) \\
& \dot{\hat{\Phi}}_{2}(t)=\tau_{\phi_{2} n^{*}}(t) \\
& \dot{\hat{b}}_{0}(t)=\left\{\begin{array}{cc}
\tau_{b n^{*}}(t) & \text { Case } \mathrm{I}_{b} \\
0 & \text { Case } \mathrm{II}_{b}
\end{array}\right. \\
& \tilde{\alpha}_{1}(t)=-k_{p} \sum_{j=2}^{n^{*}}\left(\frac{1}{4 k_{j 1}} g_{3}^{2}+\frac{1}{4 k_{j 2}} g_{4}^{2}+\frac{1}{4 k_{j 3}} g_{4}^{2}\right) z_{1}(t)
\end{aligned}
$$$$
\tilde{\alpha}_{i}(t)=-k_{i 1} \gamma_{p i-1}(t)^{2} v_{0}(t)^{2} z_{2}(t)
$$$$
-k_{i 2} \gamma_{b i-1}(t)^{2} v_{1}(t)^{2} z_{i}(t)
$$$$
-k_{i 3} \gamma_{b i-1}(t)^{2} z_{i}(t)^{2} z_{i}(t)
$$$$
-\left(n^{*}-1\right) k_{i 4} \gamma_{b i-1}(t)^{2} u_{f n^{*}-1}(t)^{2} z_{i}(t)
$$$$
-\sum_{j=2}^{n^{*}} \frac{g_{4}^{2}}{4 k_{j 4}} \gamma_{i-1}(t)^{2} z_{i}(t)
$$$$
-\sum_{j=2}^{i-1} \gamma_{\phi_{1} j-1}(t) G_{1} \gamma_{i-1}(t) \omega_{1}(t) z_{j}(t)
$$$$
-\sum_{j=2}^{i-1} \gamma_{\phi_{2} i-1}(t) G_{2} \gamma_{i-1}(t) \omega_{2}(t) z_{j}(t)
$$$$
\left(2 \leq i \leq n^{*}\right)
$$

ただし $k_{j 1} \sim k_{j 4}>0$ で $k_{p} \geq M_{p}>0\left(k_{p} b_{0} \geq 1\right)$ とする. また Case $\mathrm{I}_{b}, \mathrm{II}_{b}$ は次のように定める.

Case $\mathrm{I}_{b}: \delta_{b}<\hat{b}_{0}<M_{b}$, または $\hat{b}_{0}=\delta_{b} \& \tau_{b n^{*}}(t) \geq 0$,

$$
\text { または } \hat{b}_{0}=M_{b} \& \tau_{b n^{*}}(t) \leq 0
$$

Case $\mathrm{II}_{b}$ : その他の場合

この時, 以下の関係が得られる [17].

$$
\left\{\hat{b}_{0}(t)-\underline{b}_{0}^{*}\right\}\left\{\dot{\hat{b}}_{0}(t)-\tau_{b n^{*}}(t)\right\} / g_{4} \leq 0
$$


また $\sigma_{i}\left(\left\|\hat{\Phi}_{i}\right\|\right)$ は次の条件を満足する $n^{*}-2$ 階連続微分 可能な正值の関数とする $\left(i=1,2, \sigma_{i 0}>0\right)$.

$$
\sigma_{i}\left(\left\|\hat{\Phi}_{i}\right\|\right)= \begin{cases}0 & \left\|\hat{\Phi}_{i}\right\| \leq M_{i} \\ \in\left[0, \sigma_{i 0}\right] & M_{i} \leq\left\|\hat{\Phi}_{i}\right\| \leq 2 M_{i} \\ \sigma_{i 0} & 2 M_{i} \leq\left\|\hat{\Phi}_{i}\right\|\end{cases}
$$

ただし $\left\|\Phi_{i}^{*}\right\| \leq M_{i}$ とする．このとき次式が成立する [17].

$$
\begin{aligned}
& \sigma_{i}\left(\left\|\hat{\Phi}_{i}\right\|\right)\left(\Phi_{i}^{*}-\hat{\Phi}_{i}\right)^{\mathrm{T}} \hat{\Phi}_{i} \\
& \quad \leq-\frac{\sigma_{i 0}}{2}\left\|\Phi_{i}^{*}-\hat{\Phi}_{i}\right\|^{2}+\frac{\sigma_{i 0}}{2}\left(\left\|\Phi_{i}^{*}\right\|+2 M_{i}\right)^{2} \\
& \sigma_{i}\left(\left\|\hat{\Phi}_{i}\right\|\right)\left(\Phi_{i}^{*}-\hat{\Phi}_{i}\right)^{\mathrm{T}} \hat{\Phi}_{i} \leq 0
\end{aligned}
$$

また次の評価より

$$
\begin{aligned}
|\dot{\hat{p}}(t)| \leq & \left|g_{3} v_{0}(t) z_{1}(t)\right| \\
|| \hat{\hat{b}}_{0}(t) & \leq\left|g_{4} v_{1}(t) z_{1}(t)\right|+\left|g_{4} z_{1}(t) z_{2}(t)\right| \\
& +\sum_{j=2}^{n^{*}}\left|g_{4} \gamma_{j-1}(t) u_{f n^{*}-1}(t) z_{j}(t)\right|
\end{aligned}
$$

$\tilde{\alpha}_{i}(t)$ を含む以下の関係が得られる $\left((86)\right.$ 式の $\tilde{\alpha}_{i}$ は次式 が成立するように選んだ）。

$$
\begin{aligned}
& -\sum_{j=2}^{n^{*}} \gamma_{p j-1}(t) \dot{\hat{p}}(t) z_{j}(t)-\sum_{j=2}^{n^{*}} \gamma_{b j-1}(t) \dot{\hat{b}}_{0}(t) z_{j}(t) \\
& \quad+\sum_{j=2}^{n^{*}} \gamma_{\phi_{1} j-1}(t)\left\{\tau_{\phi_{1} j}(t)-\dot{\Phi}_{1}(t)\right\} z_{j}(t) \\
& \quad+\sum_{j=2}^{n^{*}} \gamma_{\phi_{2} j-1}(t)\left\{\tau_{\phi_{2} j}(t)-\dot{\Phi}_{2}(t)\right\} z_{j}(t) \\
& \quad+\sum_{j=2}^{n^{*}} \tilde{\alpha}_{j}(t) z_{j}(t) \leq 0
\end{aligned}
$$

以上をまとめると $V_{i}(t)$ に関して最終的に次の評価式が 導かれる。

$$
\begin{aligned}
& \sum_{i=1}^{n^{*}} \dot{V}_{i}(t) \leq r_{1}\left\{\frac{\hat{b}_{0}(t) z_{1}(t)}{2 r_{1}}+v_{1}(t)\right\}^{2} \\
& \quad-\left\{h_{1} z_{1}(t)^{2}+r_{1} v_{1}(t)^{2}\right\} \\
& \quad-\gamma_{1}^{* 2}\left\|\Delta-\frac{1}{2 \gamma_{1}^{* 2}} \Omega_{1}(t)\left|z_{1}(t)\right|\right\|^{2}+\gamma_{1}^{* 2}\|\Delta\|^{2} \\
& \quad+\tilde{b}_{0}(t) v_{1}(t) z_{1}(t) \\
& \quad+\sum_{i=2}^{n^{*}}\left[r_{i}\left\{\frac{z_{i}(t)}{2 r_{i}}+v_{i}(t)\right\}^{2}\right. \\
& \quad-\left\{h_{i} z_{i}(t)^{2}+r_{i} v_{i}(t)^{2}\right\} \\
& \left.\quad-\gamma_{i}^{* 2}\left\|\Delta-\frac{1}{2 \gamma_{i}^{* 2}} \Omega_{i}(t)\left|z_{i}(t)\right|\right\|^{2}+\gamma_{i}^{* 2}\|\Delta\|^{2}\right] \\
& \quad+\sum_{j=1}^{2} \sigma_{j}\left(\left\|\hat{\Phi}_{j}\right\|\right)\left\{\Phi_{j}^{*}-\hat{\Phi}_{j}(t)\right\}^{\mathrm{T}} \hat{\Phi}_{j}(t)
\end{aligned}
$$

ただし $\tilde{b}_{0}(t) v_{1}(t) z_{1}(t) \leq 0$ に注意する。これより本論文 の主要結果が得られる（証明は $\sum_{i=1}^{n^{*}} V_{i}(t)$ の評価式 $(94)$
式と (90)式より [13] と同様にして行うことができる).

【定理 1】 以上の適応制御系 $\left(v_{1}^{*}(t) \sim v_{n^{*}}^{*}(t)\right.$ も含む) は一様有界となる.またとくに本論文の適応則を用いな くても, 任意の有界な $\hat{b}_{0}(t), \hat{p}(t), \hat{\Phi}_{2}(t), \hat{\Phi}_{2}(t)$ (一定值 でもよい) に対して制御系は有界となる。

【定理 2】以上の適応制御系において $b_{0} \equiv$ const.の場 合を考える。このとき適応制御系は一様有界で, $v_{1}^{*}(t) \sim$ $v_{n^{*}}^{*}(t)$ はそれ以外の部分から定まる制御系に対して, 次 の評価関数の上界を最小化する準最適制御入力となる.

$$
\begin{aligned}
J(t) & \equiv \sup _{\Delta \in \mathcal{L}_{2}}\left[\sum_{i=1}^{n^{*}} \int_{0}^{\mathrm{T}}\left\{h_{i} z_{i}(\tau)^{2}+r_{i} v_{i}(\tau)^{2}\right\} d \tau\right. \\
& \left.+\sum_{i=1}^{n^{*}} V_{i}(t)-\sum_{i=1}^{n^{*}} \gamma_{i}^{* 2} \int_{0}^{\mathrm{T}}\|\Delta(\tau)\|^{2} d \tau\right]
\end{aligned}
$$

また次式も成立する。

$$
\begin{aligned}
& \sum_{i=1}^{n^{*}} \int_{0}^{\mathrm{T}}\left\{h_{i} z_{i}(\tau)^{2}+r_{i} v_{i}(\tau)^{2}\right\} d \tau+\sum_{i=1}^{n^{*}} V_{i}(t) \\
& \leq \sum_{i=1}^{n^{*}} V_{i}(0)+\sum_{i=1}^{n^{*}} \gamma_{i}^{* 2} \int_{0}^{\mathrm{T}}\|\Delta(\tau)\|^{2} d \tau
\end{aligned}
$$

とくに $\mu_{1}=\mu_{2}=d_{1}=d_{2}=0, \Phi_{i}-\Phi_{i}^{*} \in \mathcal{L}_{2}(i=1,2)$ の 時には, $z_{1}(t) \sim z_{n^{*}}(t) \rightarrow 0$ が達成される.

【定理 3】以上の適応制御系において $b_{0} \neq$ const.の 場合を考える。このとき適応制御系は一様有界で次式が 成立する。

$$
\begin{aligned}
& \sum_{i=1}^{n^{*}} \int_{0}^{\mathrm{T}}\left\{h_{i} z_{i}(\tau)^{2}+r_{i} v_{i}(\tau)^{2}\right\} d \tau+\sum_{i=1}^{n^{*}} V_{i}(t) \\
& \quad+\int_{0}^{\mathrm{T}} \frac{\tilde{b}_{0}(\tau) \hat{b}_{0}(\tau)}{2 r_{1}} z_{1}(\tau)^{2} d \tau \\
& \leq \sum_{i=1}^{n^{*}} V_{i}(0)+\sum_{i=1}^{n^{*}} \gamma_{i}^{* 2} \int_{0}^{\mathrm{T}}\|\Delta(\tau)\|^{2} d \tau
\end{aligned}
$$

とくに $\mu_{1}=\mu_{2}=d_{1}=d_{2}=0, \Phi_{i}-\Phi_{i}^{*} \in \mathcal{L}_{2}(i=1,2)$ の 時には, $z_{1}(t) \sim z_{n^{*}}(t) \rightarrow 0$ が達成されると同時に, 定理 2 の準最適制御系に漸近的に収束する。

【定理 4】特定の $\Phi_{i}^{*}, b_{0}^{*}, p^{*}\left(b_{0}^{*} p^{*}=1\right)$ に対して, $\left(\Phi_{i}-\right.$ $\left.\Phi_{i}^{*}\right),\left(b_{0}-b_{0}^{*}\right),\left(p-p^{*}\right) \in \mathcal{L}_{2}$, および $b_{0} \rightarrow b_{0}^{*}, p \rightarrow p^{*}, \mu_{1}=$ $\mu_{2}=d_{1}=d_{2}=0$ とする. この時, $z_{1}(t) \sim z_{n^{*}}(t) \rightarrow 0$ (as $t \rightarrow \infty)$ が成立する.

つぎに $h_{i}$ と $r_{i}$ を特定の形式に限定することで，それ らと制御器の陽な表現を求める.

【解法 I】 (49) (78) 式より $r_{i}(i \geq 1)$ は次のように選 ベる.

$$
\begin{aligned}
& \frac{1}{r_{i}}=\frac{k_{i 5}+k_{i 6}\left\|\Omega_{i}\right\|^{2}}{r_{0 i}} \Leftrightarrow r_{i}=\frac{r_{0 i}}{k_{i 5}+k_{i 6}\left\|\Omega_{i}\right\|^{2}} \\
& \left(k_{i 5}, k_{i 6}, r_{0 i}>0\right)
\end{aligned}
$$

ただし $k_{i 5}, k_{i 6}, r_{0 i}$ は設計変数である.これより $h_{1}, h_{i}(i \geq$ 
2)は

$$
\begin{aligned}
& h_{1} \leq \frac{\left\{\hat{b}_{0}^{2} k_{15} \gamma_{1}^{* 2}+\left(\hat{b}_{0}^{2} k_{16} \gamma_{1}^{* 2}-r_{01}\right)\left\|\Omega_{1}\right\|^{2}\right\}}{4 r_{01} \gamma_{1}^{* 2}} \\
& h_{i} \leq \frac{\left[k_{i 5} \gamma_{i}^{* 2}+\left(k_{i 6} \gamma_{i}^{* 2}-r_{0 i}\right)\left\|\Omega_{i}\right\|^{2}\right]}{4 r_{0 i} \gamma_{i}^{* 2}} \quad(i \geq 2)
\end{aligned}
$$

となり, $h_{1}, h_{i}(i \geq 2)$ が正定となるための $k_{16}$ と $k_{i 6}$ の 条件式は次のようになる。

$$
\begin{aligned}
& k_{16} \geq \frac{r_{01}}{\hat{b}_{0}^{2} \gamma_{1}^{* 2}}\left(\Leftarrow k_{16} \geq \frac{r_{01}}{\delta_{b}^{2} \gamma_{1}^{* 2}}\right),\left(0<\delta_{b} \leq b_{0}, \hat{b}_{0}\right) \\
& k_{i 6} \geq \frac{r_{0 i}}{\gamma_{i}^{* 2}},(i \geq 2)
\end{aligned}
$$

また制御入力は以下の式で与えられる.

$$
\begin{aligned}
& v_{1}=-\frac{\hat{b}_{0}}{2 r_{1}} z_{1}=-\frac{\hat{b}_{0}\left[k_{15}+k_{16}\left\|\Omega_{1}\right\|^{2}\right]}{2 r_{01}} z_{1} \\
& v_{i}=-\frac{1}{2 r_{i}} z_{i}=-\frac{k_{i 5}+k_{i 6}\left\|\Omega_{i}\right\|^{2}}{2 r_{0 i}} z_{i} \quad(i \geq 2)
\end{aligned}
$$

【解法 II】 以下のような制約をおいて $r_{i}$ と $h_{i}$ を求 める.

$$
\begin{aligned}
& h_{1}=a_{1} r_{11}+\frac{\hat{b}_{0}^{2}}{4 r_{10}} \\
& h_{i}=a_{i} r_{i 1}+\frac{1}{4 r_{i 0}}(i \geq 2) \\
& \frac{1}{r_{i}}=\frac{1}{r_{i 0}}+\frac{1}{r_{i 1}}
\end{aligned}
$$

たたし $a_{i}, r_{i 0}\left(0<a_{i}, r_{i 0}<\infty\right)$ は, $r_{i}$ と $h_{i}$ の関係を規定 する正の定数である。このとき (49) (78) 式の等号条件 で $r_{i 1}$ が以下の 2 次方程式の正值解として求められる.

$$
\begin{aligned}
& a_{1} r_{11}^{2}+G_{1} r_{11}-\frac{\hat{b}_{0}^{2}}{4}=0 \\
& G_{1}=\frac{\left\|\Omega_{1}\right\|^{2}}{4 \gamma_{1}^{* 2}} \\
& a_{i} r_{i 1}^{2}+G_{i} r_{i 1}-\frac{1}{4}=0(i \geq 2) \\
& G_{i}=\frac{\left\|\Omega_{i}\right\|^{2}}{4 \gamma_{i}^{* 2}}
\end{aligned}
$$

これより $r_{i 1}$ と $h_{i}$ は次のようになり，

$$
\begin{aligned}
& r_{11}=\frac{-G_{1}+\sqrt{G_{1}^{2}+a_{1} \hat{b}^{2}}}{2 a_{1}}=\frac{\hat{b}_{0}^{2}}{2\left\{\sqrt{G_{1}^{2}+a_{1} \hat{b}_{0}^{2}}+G_{1}\right\}} \\
& r_{i 1}=\frac{-G_{i}+\sqrt{G_{i}^{2}+a_{i}}}{2 a_{i}}=\frac{1}{2\left\{\sqrt{G_{i}^{2}+a_{i}}+G_{i}\right\}} \\
& h_{1}=\frac{-G_{1}+\sqrt{G_{1}^{2}+a_{1} \hat{b}_{0}^{2}}}{2}+\frac{\hat{b}_{0}^{2}}{4 r_{10}} \\
& h_{i}=\frac{-G_{i}+\sqrt{G_{i}^{2}+a_{i}}}{2}+\frac{1}{4 r_{i 0}}(i \geq 2)
\end{aligned}
$$

制御入力は次式で表される。

$$
\begin{gathered}
v_{1}=-\frac{\hat{b}_{0}}{2 r_{1}} z_{1} \\
=-\left\{\frac{1}{\hat{b}_{0}}\left(\sqrt{G_{1}^{2}+a_{1} \hat{b}_{0}}+G_{1}\right)+\frac{\hat{b}_{0}}{2 r_{10}}\right\} z_{1} \\
v_{i}=-\frac{1}{2 r_{i}} z_{i}=-\left\{\left(\sqrt{G_{i}^{2}+a_{i}}+G_{i}\right)+\frac{1}{2 r_{i 0}}\right\} z_{i} \\
(i \geq 2)
\end{gathered}
$$

二つの解法で求めた制御器に対して, 次の定理が成 り立つ（証明は $\sum_{i=1}^{n^{*}} V_{i}(t)$ の評価式 $(94)$ 式と $(90)$ 式よ り [13]と同様にして行うことができる).

【定理 5】解法 I または解法 II 用いると, 設計変 数 $k_{i 5}, k_{i 6}, r_{0 i}, a_{i}, \gamma_{i}^{*}$ の適切な選択により(十分大きな $k_{i 5}, k_{i 6}, a_{i}$ と十分小さな $\left.r_{0 i}, \gamma_{i}^{*}\right), z_{i}$ の残差領域を任意 に小さくできる。

\section{5. 数值例}

本手法の有効性を確認するために相対次数が 2 次の場 合について数值実験を行った. 以下の追值制御問題を考 える.

$$
\begin{aligned}
& \dot{y}(t)=f(y(t))+u_{f}(t) \\
& \dot{u}_{f 1}(t)=-\lambda u_{f}(t)+u(t) \\
& e(t)=y(t)-r(t) \\
& f(y)=y+2 \sin y \\
& r(t)=\sin t
\end{aligned}
$$

ここに $f(y)$ が以下のようにニューラルネットで近似で きるとして適応制御系を構成した。

$$
\begin{aligned}
& f(y)=W^{\mathrm{T}} S\left(V^{\mathrm{T}} \bar{Z}\right)+\mu \\
& \bar{Z}=[y, 1]^{\mathrm{T}},|\mu| \leq d, m=5
\end{aligned}
$$

設計変数は次のように定めた。

$$
\begin{aligned}
& g=100 \text { (適応ゲイン) } \\
& M=100(\sigma \text { - 修正則のパラメータ }), \quad \lambda=1 \\
& k_{i j}=5, r_{10}=r_{20}=1, a_{1}=a_{2}=1, \gamma_{1}^{*}=\gamma_{2}^{*}=0.1 \\
& \gamma=0.01 \text { (シグモイド関数のパラメータ }) \\
& \sigma_{0}=0.1 \\
& \sigma\left(\left\|\hat{\Phi}_{i}\right\|\right)= \begin{cases}0 & \left\|\hat{\Phi}_{i}\right\| \leq M \\
\sigma_{0}\left(\frac{\left\|\hat{\Phi}_{i}\right\|}{M}-1\right) & M \leq\left\|\hat{\Phi}_{i}\right\| \leq 2 M \\
\sigma_{0} & \\
2 & M \leq\left\|\hat{\Phi}_{i}\right\|\end{cases}
\end{aligned}
$$

Fig.1 と Fig.2 にそれぞれ解法 I と II を用いた結果を 示す。 


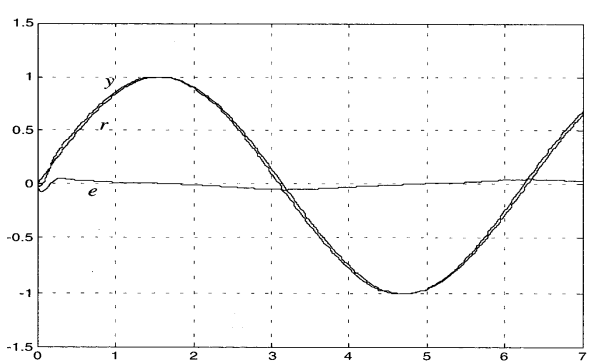

Fig. 1 Simulation result (method I)

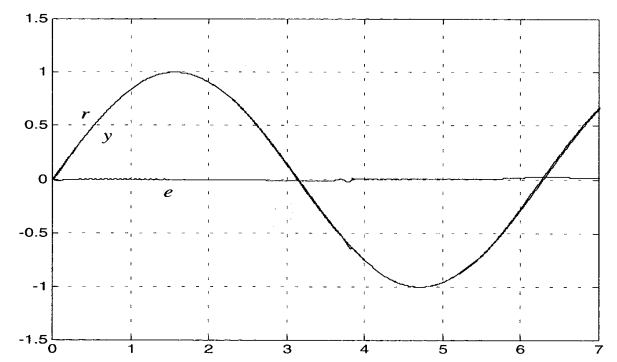

Fig. 2 Simulation result (method II)

\section{6. 結言}

システムパラメータとニューラルネットワーク（NN） に含まれる誤差を外乱と見なして，この未知外乱（パラ メータと NN の誤差）から一般化出力までの $\mathcal{L}_{2}$ ゲイン を規定するような, 非線形適応 $\mathcal{H}_{\infty}$ 制御系について述 べた。この制御方式が非線形特性やシステムパラメータ が時間変化する場合にも適用可能で制御系の有界性が保 証されること（定理 $\mathbf{1} ）$, とくに高調波利得が時不変の 時にはある $\mathcal{H}_{\infty}$ 制御問題に対する最適（または準最適） 解となっていること（定理 2 ）, パラメータが時変の場 合でもパラメータと NN の誤差から一般化出力への $\mathcal{L}_{2}$ ゲインが陽に規定されていること（定理 3 ），理想的な 条件下（パラメータが時不変または一定值に収束し NN の誤差が0）で制御誤差のゼロ収束が保証されることを 示した（定理 4）. また, 制御器の一般化形式に加えて, 二つの陽な制御器の表現も与え, 設計変数を適切に定め ると, システムパラメータの変動や NNの誤差の大きさ に関わらず，制御誤差の残差領域を任意に小さくできる ことも示した（定理 5).

\section{参考文献}

[1] M. Brown and C. Harris: Neurofuzzy Adaptive Modelling and Control, Prentice-Hall International (1994)

[2] M. M. Polycarpou and P. A. Ioannou: Neural networks as on-line approximators of nonlinear systems; Proceedings of the 31st IEEE CDC, pp. 7-12 (1992)

[3] F. C. Chen and H. K. Khalil: Adaptive control of a class of nonlinear discrete-time systems using neural networks; IEEE Trans. Automatic Control, Vol. 40, pp. 791-801 (1995)
[4] F. C. Chen and H. K. Khalil: Adaptively controlling nonlinear continuous-time systems using multilayer neural networks; IEEE Trans. Automatic Control, Vol. 39, pp. 1306-1310 (1994)

[5] T. Zhang, S. S. Ge and C. C. Hang: Design and performance analysis of a direct adaptive controller for nonlinear systems; Automatica, Vol. 35, pp. 1809-1817 (1999)

[6] J. Q. Gong and B. Yao: Neural network-based adaptive robust control of a class of nonlinear systems in normal form; Proceedings of 2000 ACC, pp. 1419-1423 (2000)

[7] A. Calise, N. Hovakimyan and H. Lee: Adaptive output feedback control of nonlinear systems using neural networks; Proceedings of 2000 ACC, pp. 3153-3457 (2000)

[8] Z. H. Li and M. Krstić: Optimal design of adaptive tracking controllers for nonlinear systems; Automatica, Vol. 33, No. 8, pp. 1459-1473 (1997)

[9] M. Krstić and H. Deng: Stabilization of Nonlinear Uncertain Systems, Springer (1998)

[10] 宮里：逆最適性に基づく非線形 $\mathcal{H}_{\infty}$ 制御; 計測と制御, Vol. 39, No. 2, pp. 112-118 (2000)

[11] 宮里: 適応 $\mathcal{H}_{\infty}$ 制御系の構成法; 計測と制御, Vol. 40, No. 10, pp. 692-697 (2001)

[12] 宮里：最適性に基づく適応制御系の再設計; 計測自動制 御学会論文集, Vol. 38, No. 9, pp. 765-774 (2002)

[13] 宮里: パラメー夕を外乱と見なした非線形適応 $\mathcal{H}_{\infty}$ 制御 系の構成法; 計測自動制御学会論文集, Vol. 39 , No. 10 , pp. 914-923 (2003)

[14] K. I. Funahashi: On the approximate realization of continuous mappings by neural-networks; Neural Networks, Vol. 2, pp. 183-192 (1989)

[15] 宮里: ニューラルネットを用いた非線形適応制御; 計測 と制御, Vol. 41, No. 12, pp. 881-887 (2002)

[16] M. Krstić, I. Kanellakopoulos and P. V. Kokotović: Nonlinear and Adaptive Control Design, John Wiley \& Suns (1995)

[17] P. A. Ioannou and J. Sun: Robust Adaptive Control, PTR Prentice-Hall (1996) 